Revue belge de géographie

\title{
La géographie sur la place : emplois, modes et modes d'emploi
}

Geography today: uses, trends and directions for use

\section{Roger Brunet}

\section{(2) OpenEdition}

\section{Journals}

\section{Édition électronique}

URL : http://journals.openedition.org/belgeo/16258

DOI : 10.4000/belgeo.16258

ISSN : 2294-9135

Éditeur :

National Committee of Geography of Belgium, Société Royale Belge de Géographie

\section{Édition imprimée}

Date de publication : 30 juin 2003

Pagination : 119-129

ISSN : 1377-2368

\section{Référence électronique}

Roger Brunet, «La géographie sur la place : emplois, modes et modes d'emploi », Belgeo [En ligne],

2 | 2003, mis en ligne le 30 juillet 2003, consulté le 19 avril 2019. URL : http://journals.openedition.org/ belgeo/16258; DOl : 10.4000/belgeo.16258

Ce document a été généré automatiquement le 19 avril 2019.

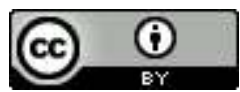

Belgeo est mis à disposition selon les termes de la licence Creative Commons Attribution 4.0 International. 


\section{La géographie sur la place : emplois, modes et modes d'emploi}

Geography today: uses, trends and directions for use

\section{Roger Brunet}

1 Préciser l'évolution de la place de la géographie dans la société sur un quart de siècle et interpréter les changements n'est pas chose facile. On a suggéré, pour orienter ces réflexions, le thème du "retour de la géographie au cœur de la société ». Je me garderai bien toutefois d'adopter une formule qui suppose qu'il existe une société définie, qu'elle a un cœur, que la géographie y est revenue - ce qui implique à la fois qu'elle y est vraiment, qu'elle y fut jadis, et qu'elle en était sortie : c'est là bien trop d'hypothèses, et qui me semblent aventureuses. C'est pourquoi je me contenterai de parler d'emplois de la géographie, de modes, et de modes d'emploi. Il ne s'agira en aucun cas d'un bilan circonstancié mais d'un témoignage, d'impressions, de vœux - avec pour horizon notre bout d'Europe.

\section{Comme un désir accru de la géographie}

Mon impression est qu'il existe pour cette période quelques indices d'une amélioration de la notoriété de la géographie, et éventuellement des géographes :

- par l'emploi même du mot, un moment assez délaissé, et qui a accru sa fréquence dans l'expression courante; il n'est plus seulement utilisé comme synonyme d'accidents de terrain, mais pour évoquer des distributions spatiales, des arrangements dans l'espace, voire des paysages ;

- dans l'emploi et l'illustration de certains concepts relevant habituellement, ou fondamentalement, de la géographie : territoire, pays, lieu et local, ainsi que Monde et Europe ;

- dans l'emploi d'outils géographiques : les cartes (y compris dans la presse et à la télévision, et même à l'Institut de la statistique, qui leur était indifférent, et même hostile, voici 20 
ans) ; les atlas de toutes sortes ; les systèmes d'information géographiques dans la pratique des collectivités locales et de l'ingénierie ; les images aériennes et satellitales - voir le succès d'Arthus-Bertrand (1999) et de recueils d'images de télédétection, pourtant quasi illisibles par le non-spécialiste ;

- dans la valorisation du «local», sensible à de nombreux indices, jusqu'à l'abondance des sites municipaux sur Internet, la multiplication des écomusées, etc. - je reviendrai sur quelques ambiguïtés en l'affaire, qui se trouvent aussi dans les points suivants ;

- dans l'emploi et la multiplication des comparaisons et classements de villes, de départements, régions ou provinces, d'États ;

- dans le nombre et le succès de manifestations s'affichant comme géographiques : le festival de Saint-Dié, la méridienne de la France, les « cafés de géographie », etc. ;

- le nombre des publications existant dans le domaine, qui a considérablement augmenté : comparez les listes actuelles aux maigres bibliographies d'antan.

3 Ces expansions - je ne dis pas nécessairement : ces progrès - sont sans doute en partie liés à des transformations $\mathrm{du}$ monde, voire des techniques. On sent comme une « géographisation » de l'ambiance.

4 La «curiosité géographique », le «sentiment géographique » comme dit Erik Orsenna (1998), ont toujours existé. Ils ont probablement profité au cours de ce quart de siècle d'un certain nombre de grands phénomènes, parmi lesquels il me semble facile de repérer :

- la généralisation et l'augmentation de rayons des voyages touristiques ;

- la mondialisation de l'information, qui vous transporte à tout moment dans des lieux attendus ou inattendus ;

- et même l'effet d'Internet, qui apparemment délocalise et en réalité fait mieux sentir l'existence des autres, et des autres lieux, dans une permanente dialectique de l'universalité et de la singularité ;

- la mondialisation tout court, qui fait prendre conscience des interdépendances et de l'unicité du Monde, surtout dans les risques évoqués, qu'il soient d'ordre écologique, ou liés à l'emploi, aux migrations, au terrorisme, à la drogue, voire à la bourse ;

- la construction de l'Europe, jusqu'à l'apparition de l'euro, qui amènent à élargir les regards ;

- la mobilité des populations et ses contre-effets de replis et d'affrontements ;

- en réaction contre ces tendances mondiales, en partie au moins, les replis sur le local, les ségrégations, les communautarismes qui jouent, parfois dangereusement, avec l'idée de territoire.

5 Il s'agit là de préoccupations, de processus et de données qui sont très loin d'être futiles, et qui ont des effets directs sur les niveaux et les modes de vie, sur les représentations et les comportements, et même sur les croyances, les idées et les idéologies.

6 L'appel à des catégories relevant de la géographie semble s'être élargi et diversifié. Nombreux sont ceux qui sont tentés d'y répondre, avec ou sans compétence. De ce fait, je me demande si la réponse des géographes eux-mêmes est vraiment à la hauteur des enjeux, et si leurs géographies ont changé autant que le monde - et autant qu'on aurait pu le souhaiter. 


\section{Cinq équations pour des fractions de société}

7 Pour éclairer cette question, il faudrait affiner. La société, c'est vague et c'est composite. Vue de plus près, elle est faite de constituants assez différents en la matière. Je vous propose de distinguer cinq catégories, à propos desquelles il est possible de formuler des hypothèses, ou des impressions, plus que des faits scientifiquement établis ; ce qui laisse la place à toutes les réévaluations et corrections que d'autres expériences imposeraient.

\section{Les jeunes, scolaires et étudiants}

Bernadette Mérenne-Schoumaker traitant de son côté de l'enseignement, je me contenterai de remarquer qu'à l'école et à l'université la géographie, à travers maintes vicissitudes, semble avoir en gros maintenu ses positions dans les pays que je connais. En quantité, elle semble avoir conservé sa place, et ses effectifs semblent avoir crû moyennement. Des régressions ou des menaces affectent parfois le domaine scolaire (comme en Italie), pas vraiment l'Université.

9 Notons dès à présent, pour ce dernier quart de siècle, un sensible élargissement des débouchés hors du monde de l'éducation; la contrepartie en est dans la difficulté où se trouvent les géographes d'alimenter suffisamment ce dernier, laissant l'enseignement de la géographie à des professeurs faiblement formés dans la discipline.

\section{Les décideurs}

10 De très sensibles progrès ont été accomplis partout dans la relation avec les décideurs de toutes sortes: élus locaux, administratifs et ingénieurs des services publics, entrepreneurs même.

11 C'est là un grand changement par rapport aux années 1970, où seuls économistes, juristes, sociologues et architectes semblaient avoir une utilité sociale hors des spécialistes de sciences naturelles. L'intrusion des géographes n'est pas unique: des anthropologues et même des historiens, voire des philosophes, ont pu être sollicités; mais elle est générale et soutenue.

12 À ma connaissance, le phénomène existe aussi en Suisse, en Espagne et en Italie; il est très sensible en Russie. Il touche tous les niveaux d'expertise: études à l'échelle européenne, nationale, régionale, locale.

13 Contrairement à ce qui se passait encore au cours des années 1960, la spécificité du travail des géographes me semble y être généralement reconnue : l'information sur les lieux, l'analyse spatiale au sens large, la cartographie et les SIG sont leurs domaines principaux de compétence.

14 Ce mouvement a permis l'ouverture de nombreux débouchés. Les jeunes géographes ont trouvé des emplois directs, par l'embauche dans les administrations et collectivités locales, parfois les entreprises, et des emplois indirects, par le recours aux bureaux d'études : quantité de géographes ont pu apparaître dans des cabinets privés, et souvent en fonder eux-mêmes. 


\section{Le milieu intellectuel} Ils ont évidemment toujours existé, mais jadis ils étaient plus souvent limités à des relations personnelles et les géographes faisaient assez volontiers bande à part dans les universités. La pratique des appels d'offre a certainement contribué à ces rapprochements; sans doute aussi les progrès accomplis par les géographes en méthodologie, en épistémologie et dans l'approche de questions de société et d'environnement y ont eu quelque part. De ce fait, et par l'adoption d'éléments de langage commun (logique et mathématique notamment), davantage de passerelles ont pu être établies et consolidées. et dans la considération et l'estime réciproques des chercheurs de différentes disciplines, cela ne semble pas s'être accompagné d'un changement fondamental du statut de la géographie dans les sciences. On n'observe pas d'entrée significative de géographes dans les débats d'idées, guère de citations significatives d'ouvrages de fond écrits par des géographes - lesquels ouvrages existent pourtant. Les références restent plutôt rares chez les philosophes, les économistes, comme d'ailleurs, semble-t-il, dans les sciences naturelles.

\section{Les médias}

Les médias se sont intéressés, davantage qu'auparavant, aux travaux de géographes, et s'y intéressent sporadiquement. Des géographes sont régulièrement consultés par la presse écrite, la radio et même la télévision, au moins dans le cadre régional, parfois au niveau national. Quelques initiatives ont eu en France une assez bonne couverture (Festival de Saint-Dié, Gip Reclus). Périodiquement, la presse découvre ou redécouvre des cartes qui excitent l'esprit (ex. certaines structures de l'espace européen, ou plus récemment des cartes de la santé et de la mortalité) - voire des phénomènes géographiques permanents mais régulièrement oubliés et sur lesquels des journalistes croient devoir aussitôt relancer des géographes (ex. le débit de l'Yonne et de la Seine, le niño, la mousson, l'énorme population de la Chine et de l'Inde, la particularité des détroits ou le sida en Afrique...). La curiosité géographique se manifeste souvent à l'occasion d'événements ; mais des géographes ont parfois su créer l'événement - surtout par la carte -, passant ainsi d'une position passive à une attitude plus active.

Il reste que, dans ces domaines, on doit prêter attention à trois points. D'abord, les géographes publient rarement des articles de fond sur des sujets majeurs dans la " grande presse ». Ensuite, le milieu des médias a sa propre existence et sa propre logique, vit en grande partie en autarcie et s'intéresse d'abord à lui-même : en dehors d'exceptions de quelques « savants » décidés à jouer tout à fait leur jeu, jusqu'à présider des concours de beauté, on a peu à en attendre - même Pierre Bourdieu s'en est plaint. Enfin, les journalistes font leur travail, avec leurs moyens propres ; l'actualité, qui est leur champ de travail, est souvent très "géographique "; or son traitement médiatique n'implique nullement le recours à des « experts »- lesquels ont d'ailleurs le plus souvent tendance à ne s'exprimer qu'avec un luxe de précautions et de circonlocutions incompatible avec le style médiatique. Il est donc assez naturel que les journalistes s'estiment autosuffisants 
sur une grande partie de notre propre champ, et nous ne saurions nous en offusquer. À nous de nous exprimer de notre côté, le cas échéant ; mais à chacun son métier : il serait imprudent de vouloir jouer au journaliste.

19 En tout cas, même si les médias ont plus souvent que jadis recours aux géographes, on ne connaît pas encore un seul géographe « médiatique » en Europe occidentale, ni sans doute dans le monde, au sens où le sont quelques philosophes, historiens, économistes, sémiologues, paléontologues, astronomes ou autres - récurrents et, en vérité, fort peu nombreux.

\section{Grand public et dirigeants politiques}

Une dernière constatation est que la géographie scientifique a peu progressé dans la conquête de ce que l'on appelle en général le grand public. La multiplication des titres d'ouvrage et la permanence des revues ne déborde guère du public universitaire ; on n'a pas vu non plus d'essai à grand tirage signé par un géographe.

Il y a eu quelques tirages à succès, mais déjà anciens (Découvrir la France a atteint 300000 ex. en fascicules hebdomadaires et albums, il y a déjà trente ans), et un succès d'édition qui revient à un géographe (Jean Malaurie), mais pour des ouvrages d'ethnographie, et dont le succès est tout aussi ancien même s'il est nettement plus durable. Seuls des atlas généraux et des annuaires parviennent aux gros tirages.

Pourtant le grand public consomme à sa façon « de la géographie ». Nul ne sait ce qu'est le grand public, mais il a ses habitudes, en partie façonnées par les médias. Si j'en rapproche les dirigeants politiques, c'est que ceux-ci aiment bien en général être en résonance avec la masse des électeurs, et les flatter dans le sens du poil. Or s'il est quelque chose qui n'a nullement changé en 25 ans, en dépit de tous nos efforts pour élever le niveau de l'information et de la réflexion du grand public et des dirigeants, c'est bien dans ce domaine.

Le discours populaire sur « la géographie » est en général cohérent, et n'a absolument pas évolué : il maintient ce que la géographie de tradition a de plus conservateur, pour ne pas dire de plus réactionnaire. Tout ce dont les géographes scientifiques s'évertuent dans le même temps à montrer l'inanité, à savoir le déterminisme naturaliste le plus grossier, la survalorisation des "racines", les "vocations" locales assignées de toute éternité, affleure dans les propos de comptoir et dans les discours publics, qui sont assez souvent du même tonneau. Au contraire, les inquiétudes, en partie légitimes, concernant certains aspects de l'évolution de la planète ont renforcé une tendance spontanée au réenchantement de la nature.

De sorte que l'image «instinctive » de la géographie, si j'ose cette expression, n'a pas changé. Cette constatation m'amène à réfléchir à « ce qui marche » en géographie, c'està-dire à ce qui est socialement admis, pour ainsi dire "socialisé "- à des degrés évidemment différents selon les catégories mêmes que je viens d'évoquer.

\section{Géographie Janus : deux versants du métier}

Je suggérerai volontiers que "la géographie qui marche", la géographie la mieux socialisée, a une double face, deux versants actifs, un ubac et un adret ou, comme l'on dit mieux dans les Pyrénées, une ombrée et une soulane. 

«La géographie » c'est ce qui est là, immuable, la nature-mère qui commande et que l'on ne doit pas trahir. Les montagnes, les rivières, les sols. On veut bien y ajouter des frontières et des villes, mais stables. Plus quelques indigènes, aussi étranges que possible : les autres ne seraient pas «authentiques ». Feuilletez les revues à grand succès qui se réclament explicitement de la géographie : Geo, et le National Geographic, qui a désormais une édition en français. Leur succès ne se dément pas. De belles photos, certes; mais de quoi ? D'animaux, de végétaux, de reliefs ; plus des «primitifs ", pourvu qu'ils aient l'air tout aussi «naturels»; quelques monuments pour varier, mais fondus dans le paysage. Quant à la vie et aux œuvres de l'immense majorité des humains, ce n'est pas l'objet. Notez d'ailleurs que ces magazines, œuvres de journalistes, se passent parfaitement des géographes de métier. "impose», jusque dans les charcutages électoraux, comme l'assurait sans rire un ministre français de l'intérieur; et les stratèges sont supposés mener leurs troupes conformément à ce terrain. "La géographie ", évidemment incontournable, est l'excuse des mauvais chefs. Certes, il n'y a pas que du faux ou des illusions en l'affaire. C'est à nous d'y réfléchir. Mais comment oublier tous les acteurs des sociétés humaines, et la complexité de leurs stratégies entremêlées, comment oublier l'invention, plus le hasard? On peut répondre que, de toute façon, toute connaissance est un gain et que, de la fréquentation accrue de ce versant ombreux, nous géographes devrions nous réjouir. Je n'en suis pas si sûr. Je ne suis pas convaincu que la contemplation des accidents de la nature porte à l'amour de l'humanité, et qu'un regard amusé et condescendant sur d'aussi étranges indigènes conduise nécessairement à l'altruisme. En revanche, j'inclinerais volontiers à penser que l'insistance sur la nature amène non seulement à vouloir la réenchanter, ce qui n'est qu'affaire de croyance personnelle, mais à s'opposer instinctivement à tout ce qui l'altère : au changement, et à l'aménagement.

Or l'humanité n'a progressé qu'en s'« arrachant » à la nature, les sociétés ont passé leur temps à la modifier, à l'aménager, à la corriger à leur convenance, au moins depuis que certaines ont inventé l'agriculture. On peut à ce sujet saluer l'ouvrage récent de Jared Diamond, De l'inégalité parmi les sociétés, paru en 1997 et traduit en français en $2000 \mathrm{chez}$ Gallimard - en regrettant au passage qu'il soit l'œuvre d'un médecin physiologiste, et non d'un géographe. Du moins est-ce un livre qui fait une large place à la géographie prise au sens large, parfois d'ailleurs presque trop, dans sa recherche des bifurcations et des inventions qui ont transformé et différencié l'humanité, altérant quelque peu la nature tout en exploitant ses propres différences.

Qu'il y ait à redire sur la façon dont certaines sociétés ont procédé à cette altération, et continuent à le faire avec les moyens surpuissants d'aujourd'hui, est un autre sujet capital, mais qui, précisément, se situe sur l'autre versant de la géographie. Car il existe un adret, une autre géographie "qui marche", dans les deux sens du mot : qui fonctionne et qui intéresse. Il est possible d'en définir quelques caractéristiques. Selon l'expérience que $j$ 'en ai et les observations que l'on peut faire :

- elle tire parti d'images fortes du territoire, qu'elle dévoile et qu'elle interprète, des images permettant de se situer, de situer les problèmes, les risques, les diffusions et d'évaluer leur ampleur : ces images qui font penser représentent par exemple les inégalités du monde, la mortalité par alcoolisme en France, la dorsale européenne, les inégalités du peuplement ou de la natalité, ou même l'extension de la rage, les chemins de la drogue, etc. ;

Belgeo, 2 | 2003 
- elle insiste sur l'analyse des différences, des disparités, des ruptures, des lignes de force, des solidarités dans l'espace mondial, continental, national ou local; toutes les formes de « rugosités » des territoires, et pas seulement les accidents du terrain ;

- elle propose aux entrepreneurs et aux élus locaux des évaluations prospectives de territoires, selon des hypothèses de développement, au sein desquelles le géographe n'a pas à conseiller, mais à analyser, et à esquisser des probabilités en fonction d'options choisies, et en toute connaissance des structures et dynamiques des territoires, des voisinages et du système des acteurs ;

- elle contribue ainsi, en effet, à l'aide à la décision, mais pas seulement à la celle des « décideurs » au sens restreint : plus généralement, à l'aide à la réflexion sur le territoire, qui n'est pas réservée aux décideurs, mais importe aussi aux citoyens; elle peut, elle doit, être un élément de la formation et de la liberté de pensée et de décision du citoyen.

31 Cette géographie obtient ses meilleurs résultats et ses succès les plus significatifs en développant tout ce que nous avons appris de l'analyse spatiale, fondée sur une approche scientifique, consciente de ses hypothèses, des biais des informations et des techniques, et étayée par une solide théorie de la production de l'espace.

Travaillant ainsi, nous n'effacerons pas l'autre versant - pas plus que les progrès de l'astronome ne réduiront le succès de l'astrologie, car il s'agit du versant de l'impensé, de l'irrationnel. Du moins pourrions-nous veiller à ne pas le peupler à notre tour, mais au contraire à l'éclairer un peu. Or cette éventualité n'est pas aussi absurde qu'il peut sembler.

\section{Des courants et des modes, ou flotter sans sombrer}

Cette opposition de versants est évidemment une figure de style un peu simple, et n'est d'ailleurs pas propre à la géographie; elle ne doit être vue que comme un modèle de tendance, ou plutôt de constante. Elle se complique du fait que la géographie n'est pas une, et qu'elle n'est pas indépendante des fluctuations des modes intellectuelles.

Il est en effet de plus en plus difficile de parler de "la géographie » et du géographe au singulier. Au cours du dernier quart de siècle, il me semble que la géographie s'est tout à la fois diversifiée et intégrée, et que c'est là un trait majeur de sa place dans la société, pour parler simplement.

35 J'ai gardé de mon premier (et lointain) apprentissage de la géographie l'image d'un corps d'assez petite taille, homogène, corporatiste et aseptique, quelque peu éloigné des débats d'idées pourtant vifs à l'époque, disposant de peu d'ouvrages de réflexion.

D'un côté, un processus de diversification interne s'est amorcé dans les années 1960 ; il s'est poursuivi et élargi depuis. Les progrès de la géographie dite humaine, l'inégal investissement dans l'apprentissage de la mesure et de l'analyse spatiale, un effort inévitablement sélectif sur la théorie, l'accroissement considérable de la population des chercheurs, peut-être aussi un élargissement général des curiosités, y sont certainement pour quelque chose. C'est ou ce fut une libération, que je salue comme telle.

7 De l'autre côté, les géographes, mieux intégrés au travail interdisciplinaire en sciences sociales, semblent se montrer plus sensibles à l'ambiance culturelle, aux modes intellectuelles générales, voire aux débats d'idées ; plus conformes en somme - c'est peutêtre par là que l'on pourrait parler d'un « retour au cœur de la société »... 

représentation de ces changements. Je me contenterai ici de souligner certains effets de cette intégration culturelle. Nous avons connu des tendances, et des polarisations. Le «tout mathématique », le «c'est l'économie qui détermine », le «tout est politique », le «c'est le culturel qui compte». Nous recevons maintenant des sollicitations vers le «politiquement correct» à l'américaine: l'on se devrait de travailler sur la différenciation sexuelle de l'espace et sur l'espace dit domestique, comme, cela va de soi, sur la pollution et les déchets. pas mauvais en soi. C'est le calme plat qui serait redoutable. Mais il serait non moins redoutable d'être emporté dans des courants de masse, d'ailleurs éventuellement réversibles en peu de temps.

41 Pour rester dans les images physiques, je dirai qu'une science procède normalement par accrétion, et non par négations successives. Elle prend et elle retient de chaque mode ce qui marche, ce qui fait penser. Et elle ne devrait pas oublier. Avoir appris à mesurer et calculer ne doit pas être vu comme une mode éphémère dont on serait enfin débarrassé, mais comme un apprentissage nécessaire et durable. Avoir appris à évaluer le poids des représentations et des déterminants économiques, politiques et culturels dans la production de l'espace géographique n'autorise ni à oublier tel ou tel par la suite, ni à élire l'un d'eux déterminant en chef.

42 Si je parle ainsi de mode, c'est parce que j'observe que la socialisation accrue des géographes a des conséquences sur leur travail et que ces conséquences méritent d'être examinées.

43 En simplifiant beaucoup, disons que l'une des tendances fortes du présent est dans la promotion de l'individu, de la quête d'identité individuelle, dût-elle s'appuyer sur des formes de communauté. Une autre est dans la défiance à l'égard de la science, l'affirmation que tout se vaut, que la vérité est inconnaissable - hors de l'inspiration qui vous révèle l'«être-là ». Les deux courants sont les constituants principaux de ce qui s'affiche comme "postmodernisme » et prétend ainsi fossiliser la modernité. Quand des géographes s'en inspirent, cela donne des résultats curieux.

44 Les uns vous diront qu'il faut étudier la vie quotidienne des personnes, leur espace familial, leurs objets familiers; sujets dignes d'intérêt en effet ; mais tout sujet d'intérêt appelle-t-il nécessairement le concours de géographes zélés? Demandons-nous d'abord en quoi un géographe apportera à cette analyse un point de vue compétent et des méthodes originales; qu'attendrions-nous d'une "géographie» de Loft Story? La géographie est outillée pour étudier certains faits de société - de toute première importance d'ailleurs, et qui requièrent toute son attention; je ne la crois pas à sa place dans tous les sujets, et notamment dans l'approche voyeuriste des individus, ni comme contribution à l'ontologie. 

géographes avons bien un domaine commun, balisé de mots clés comme espace, territoire, pays, réseau, lieu, milieu, distance, diffusion, voisinage, etc. et je dirai même un champ propre : celui de la production et de l'organisation de l'espace terrestre - qui suppose, entre autres, quelque connaissance de la Terre elle-même, en tant que planète porteuse de vie. sociale unifiée: c'est l'étude propre d'objets de science spécifiques, avec des outils privilégiés, et une compétence qui déborde du seul sujet social, même si c'est pour mieux interpréter celui-ci. auparavant, bien entendu), et de ce que l'on peut observer de leur usage social, à travers 
réussites et carences, il me semble que l'on peut assez bien voir à quoi pourrait, sinon devrait, s'employer la géographie demain.

1. Tout d'abord à poursuivre des recherches librement décidées et librement conduites, aussi «pures» qu'on le voudra, sans souci de financement sur contrat (ce qui implique des aides financières hors de tout programme imposé) et sur n'importe quel sujet: la liberté de la recherche est comme une assurance sur l'innovation (cf. R. Brunet, 2003). Le seul souhait que je ferais volontiers serait que cette recherche soit menée selon des méthodes rationnelles et donne lieu à des publications : rendre compte de notre recherche est notre façon de rendre des comptes.

2. Tenir bon sur l'objet central, qui est l'intelligence des localisations et l'analyse spatiale. Cela suppose des travaux accrus sur les dynamiques des populations et des lieux, la division spatiale du travail, les réseaux et les ruptures, les agrégations, ségrégations et retranchements, la distribution des inégalités sociales, les mailles territoriales et la production foncière, l'écologie (au sens large), les transformations de l'environnement et les risques, les dissymétries et les effets de distance, etc.; et bien entendu des recherches sur ceux qui produisent l'espace géographique: les acteurs, leurs représentations et leurs stratégies.

3. Investir davantage dans la connaissance des mouvements des idées, afin de mieux se situer, ce qui devrait aller de soi pour un spécialiste des situations.

4. Développer notre savoir sur des thèmes scientifiques transversaux, ceux où notre apport interdisciplinaire est en général le plus apprécié :

1. la mise en situation (dans les champs, les voisinages, les écosystèmes, les réseaux, les ruptures, etc.) ;

2. la mise en perspective: les changements de point de vue, d'azimut, d'orientation dans l'observation des phénomènes ;

3. la mise en échelle et les effets des changements d'échelle des phénomènes ;

4. la mise en carte, qui intègre les précédentes en leur ajoutant une dimension synoptique

5. Etre plus assidus et plus attentifs en matière, précisément, d'information géographique. Il me semble que nous ne faisons pas assez bien notre travail de base, qui est la description géographique, celle des lieux, de leurs qualités et activités, de leurs changements. On ne peut pas laisser à la CIA le monopole de l'information géographique, et nous devons au contraire diffuser cette information à tous par nos revues, nos livres, par Internet, etc. Nous ne sommes sans doute pas libérés d'une crainte de l'inventaire, nous avons toujours peur de retomber dans une vieille géographie purement factuelle. Mais ce n'est plus de saison, puisque nous avons montré que nous savons faire tout autre chose. D'ailleurs nous n'hésitons pas devant des programmes de SIG hautement détaillés et fort coûteux, mais purement numériques et sans noms de lieux. Il nous faut reparler des pays et des lieux, avec précision.

6. Travailler et s'exprimer directement sur les grandes questions qui préoccupent nos sociétés. Et, par exemple,

1. la mondialisation et les dégâts de la loi du profit : nous avons à ce sujet trop de timidité dans l'analyse, une vision trop technicienne des relations mondiales à travers les flux ; nous devrions analyser les politiques géographiques des entreprises, tant à l'échelle mondiale qu'au niveau local, montrer la recréation permanente des différences géographiques, faire un effort vers la géographie dite sociale selon les uns, ou radicale selon d'autres ;

2. la relation des sociétés à leur environnement: la géographie a fort heureusement conservé un corps étoffé de spécialistes des questions de nature, qui ont su sortir du champ limité de la géomorphologie pour s'intéresser aux fluides et au vivant, et qui ont investi le thème des risques; il faut qu'ils se fassent mieux entendre, avec plus de hauteur 
de vue, dans un domaine où se cultivent et fleurissent les psychoses et l'irrationnel ; et qu'ils sachent évaluer dans ces domaines aussi les effets géographiques de la spéculation et de la recherche du profit à court terme ;

3. les espaces d'innovation, pionniers ou non : savoir dire et pouvoir montrer que le monde n'est pas fini, que l'humanité dispose de nombreuses ressources inexplorées, de vastes possibilités de valorisation de sols et de sites, que l'on crée toujours des villes nouvelles et même des voies ferrées, et comment le monde a changé en 25 ans dans tous ses continents ;

4. les disparités, les ségrégations, les migrations, les aspects contradictoires de la territorialisation de la vie sociale; les formes de retranchement qui divisent nos espaces familiers, et la géographie des antimondes qui élargissent ceux de l'illégalité ;

5. le local, c'est-à-dire la gestion des territoires et de l'espace social à l'échelle locale, où les géographes ont déjà mené des expériences et parfois élaboré des formes nouvelles qui permettent l'expression des citoyens eux-mêmes, et pas seulement des " décideurs » ou des experts (cf. d'Aquino et le débat de L'Espace géographique, $\mathrm{n}^{\circ} 1$ de 2002).

7. Et, oui, continuer à travailler en géographie "appliquée ", sans état d'âme et sans complaisance. Nul chercheur n'y est obligé, et l'on peut se plaire et se limiter à la recherche «pure». Nul chercheur n'est obligé de ne travailleur que pour les puissants, même s'ils financent mieux : notre savoir peut, doit être aussi au service des ONG, des associations, etc.

\section{Conclusion}

Par ces chemins et dans ces champs les géographes pourront contribuer à une théorie critique de la société. Il me semble bien qu'en 25 ans la géographie a progressé sur son adret, dans son emploi social et vers cette forme d'utilité sociale qui est le devoir de critique et de lucidité de l'intellectuel.

Néanmoins, ce qui a manqué le plus aux géographes durant ces 25 ans, ce n'est pas la science, même approximative parfois; ce n'est pas l'information, même si les données statistiques ne sont pas partout satisfaisantes ; ce n'est pas l'emploi, le débouché ; ce n'est même pas le financement et le travail sous contrat; c'est d'oser se prononcer sur de grandes questions qui sont au moins en partie de nos compétences, en cultivant quelque peu l'art de s'exprimer.

55 Mon vœu est que la géographie y parvienne peu à peu, non par de hasardeuses métaphysiques et leurs facilités momentanées, mais par son versant des Lumières - au sens philosophique et historique de ce mot.

\section{RÉSUMÉS}

On peut estimer que, en un quart de siècle, les usages sociaux de la géographie ont progressé dans l'aide à la décision, l'aménagement du territoire, le recours aux cartes et aux nouveaux outillages. Développement local, construction européenne et mondialisation ont contribué, avec l'évolution des pratiques et des réflexions des géographes eux-mêmes, à cet élargissement des 
emplois de la géographie. L'image instinctive de la géographie, sa place dans le champ disciplinaire et sa position médiatique n'en ont pas été bouleversés pour autant. Les avancées dans tous ces différents domaines apparaissent d'autant plus nettes qu'elles s'appuient sur la rigueur scientifique, l'ouverture aux autres sciences et la conscience claire de la spécificité du travail des géographes, plutôt que sur les aspects les plus convenus des modes culturelles et des discours associés. C'est en travaillant sur l'information géographique, l'analyse spatiale, la réflexion sur les effets de situation, de perspective et d'échelle, en approfondissant l'étude des différences sociales et spatiales et des problèmes d'environnement que la géographie peut attendre de nouveaux emplois et rendre des services mieux appréciés.

It can be deemed that, within a quarter of a century, the social uses of geography have advanced decision aid, territorial planning, the use of maps and new tools. Local development, European construction, globalisation, along with the evolution of the practices and reflections of geographers themselves, have contributed to multiply the uses of geography. Yet the instinctive image of geography, its place within the disciplinary field and its mediatic position have not changed for all that. The progresses in those fields appear all the more obvious as they are based on scientific rigour, opening to other disciplines and a clear awareness of the specificity of the geographers' work, rather than on the most conventional aspects of cultural fashions and related discourses. It is through working on geographical information, spatial analysis, reflection on location, perspective and scale effects, and through improving the study of social and spatial differences and of environmental issues that geography can expect increased and improved uses.

\section{INDEX}

Mots-clés : usages de la géographie, mondialisation, modes scientifiques, image géographique

Keywords : uses of geography, globalisation, scientific fashions, geographical image

\section{AUTEUR}

\section{ROGER BRUNET}

Professeur des Universités, Directeur de recherche émérite 